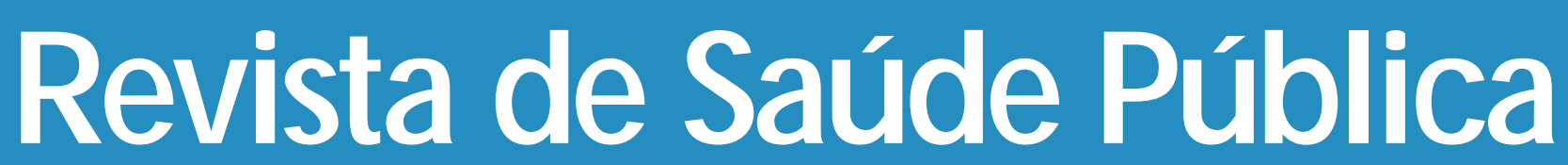

$\begin{array}{lllllll}J & 0 & \mathbf{U} & \mathbf{R} & \mathbf{N} & \mathbf{A} & \mathbf{L}\end{array}$

O F

$\begin{array}{llllll}P & \mathbf{U} & \mathrm{B} & \mathrm{L} & \mathbf{I} & \mathrm{C}\end{array}$

H E A L T H

\title{
Incidência e duração da amamentação conforme o tipo de parto: estudo longitudinal no Sul do Brasil*
}

\section{Incidence and duration of breast-feeding by pattern of delivery: a longitudinal study in Southeastern Brazil}

Elisabete Weiderpass, Fernando C. Barros, Cesar G. Victora, Elaine Tomasi e Ricardo Halpern Departamento de Medicina Social da Faculdade de Medicina da Universidade Federal de Pelotas. Pelotas, RS - Brasil

W eiderpass Elisabete Incidência e duração da amamentação conforme o tipo de parto: estudo longitudinal no Sul do Brasil* Rev. Saúde Pública, 32 (3): 225-31, 1998

(c) Copyright Faculdade de Saúde Pública da U SP. Proibida a reprodução mesmo que parcial sem a devida autorização do Editor Científico. Proibida a utilização de matérias para fins comerciais. All rights reserved. 


\title{
Incidência e duração da amamentação conforme 0 tipo de parto: estudo longitudinal no Sul do Brasil*
}

\section{Incidence and duration of breast-feeding by pattern of delivery: a longitudinal study in Southeastern Brazil}

\author{
Elisabete Weiderpass, Fernando C. Barros, Cesar G. Victora, Elaine Tomasi e Ricardo \\ Halpern \\ Departamento de Medicina Social da Faculdade de Medicina da Universidade Federal de Pelotas. \\ Pelotas, RS - Brasil
}

\begin{abstract}
Resumo
Objetivo

Comparar a incidência de amamentação conforme o tipo de parto.

Material e Método

Resultados

Conclusões

Objective

Methodology

Results

Estudo de coorte prospectivo de base populacional com 655 crianças brasileiras nascidas no ano de 1993 e acompanhadas durante o primeiro trimestre de vida, através de duas visitas domiciliares.

A duração da amamentação foi similar entre os nascidos por parto vaginal e cesariana emergencial. Os nascidos por cesariana eletiva apresentaram um risco três vezes maior de interromper a lactação no primeiro mês de vida, após controle de fatores de confusão (razão de odds=3,09; 95\% IC 1,3-7,2). Este aumento de risco não persitiu até o terceiro mês de vida.

Recomenda-se aos serviços de saúde atenção especial a lactantes submetidas a cesarianas eletivas, para que seja promovido o aleitamento desde as primeiras semanas, evitando a interrupção precoce.

Aleitamento materno. Cesárea. Parto normal.

\section{Abstract}

To compare the incidence of breastfeeding according to type of delivery.

Population-based cohort study of 655 children born in 1993 and followed up during the first three months of life through home visits.

Breastfeeding duration was similar among babies born either by vaginal delivery or by emergency cesarean section. Babies born by elective cesarean section, however, presented a three times higher risk of stopping brestfeeding in the first month of life, after adjusting for possible confounding factors (odds ratio=3.09; 95\% CI 1.3-7.2). The increased risk did not persist into the third month of life.
\end{abstract}

* Subvencionado pelo Centro de Controle de Doenças Diarréicas da Organização Mundial de Saúde, pela Comunidade Econômica Européia e pela Fundação de Amparo à Pesquisa do Estado do Rio Grande do Sul (FAPERGS). 
Conclusions It is recommended that health services pay special attention, in the promotion of breastfeeding, to babies born by elective cesarean section, as a way to avoid early weaning.

Breast feeding. Cesarean section. Natural childbirth.

\section{INTRO DU ÇÃO}

O reconhecimento dos benefícios do aleitamento materno ${ }^{15}$ tem levado à busca das causas de seu insucesso freqüente e interrupção precoce. A eficiência de medidas de intervenção para promover o aleitamento depende, principalmente, da identificação de mães com risco aumentado de não iniciar ou interromper precocemente a lactação. Esse risco tem sido associado, dentre outros fatores, com o tipo de parto, particularmente com cesarianas ${ }^{9,28}$.

A proporção de cesarianas varia marcadamente entre países $^{24}$, regiões e subpopulações ${ }^{2,14,30}$. O rápido aumento de partos por cesariana ocorrido na década de 1970, na maioria dos países, deu lugar a uma desaceleração na década de $1980^{25}$. No Brasil, onde cerca de um terço dos partos ocorre por cesarianas, também verificou-se esta tendência ${ }^{3}$. Apesar de que proporções aceitáveis ainda não tenham sido definidas, considera-se bastante alto este índice, comparado com outros países ${ }^{10,17}$.

A comparação dos estudos que relataram um risco aumentado de problemas na lactação entre mães submetidas a cesarianas $4,7,11,22,23,26,27,29,31,34,37$ com os que não mostraram estes resultados 2,12,15,16,18,21,32,33,39 é dificultada pelas diferentes metodologias empregadas, por vezes inadequadas ou insuficientemente descritas. Os principais problemas detectados foram: falta de controle de fatores de confusão, imprecisão de indicadores utilizados, tamanho de amostra insuficiente para detectar a associação, altas proporções de perdas, populações em estudo submetidas a programas específicos para promoção do aleitamento materno, resultados insuficientemente descritos e limitação de amostras a um único hospital ou clínica. Se a amamentação ótima (exclusiva até 4-6 meses e parcial até o final do primeiro ano de vida) for dificultada pelas cesarianas, este tipo de parto pode ter conseqüências negativas sobre a saúde e sobrevivência infantis ${ }^{13}$.

O propósito do presente estudo é verificar o impacto do tipo de parto - vaginal, cesariana eletiva ou cesariana emergencial - sobre a incidência e a duração da lactação em uma coorte de crianças no Sul do Brasil, acompanhadas desde o nascimento até o terceiro mês de vida.

\section{MATERIAL E MÉTO DO}

O estudo perinatal das crianças nascidas em Pelotas, cidade de porte médio e predominantemente urbana no Sul do Brasil, identificou todos os 6.410 nascimentos hospitalares ocorridos no ano de 1993. Os bebês foram examinados para determinação da idade gestacional ${ }^{6} \mathrm{e}$ aferição do peso ao nascer no pós-parto imediato, durante a estadia hospitalar. Nessa ocasião, as mães foram entrevistadas por médicos ou estudantes de medicina, respondendo a questionários padronizados e pré-codificados. As questões referiam-se às características socioeconômicas, demográficas, relativas ao período pré-natal (hospitalizações maternas e frequiência a programas prénatais), história reprodutiva, morbidade do recém-nascido e tipo de parto. Em caso de parto por cesariana, a mãe respondia qual o motivo que levou à sua realização, e o entrevistador classificava esse motivo de acordo com as opções mais comumente apresentadas e definidas em uma fase-piloto do estudo. Posteriormente, esses motivos foram reclassificados em dois grupos - cesariana eletiva ou emergencial - de acordo com critério arbitrário definido pelo grupo de pesquisa (ver definições a seguir).

Optou-se pela categoria "classe social" como indicador socioeconômico por entender-se que traduz mais adequadamente do que a renda familiar a realidade brasileira contemporânea ${ }^{34}$. A classe social foi caracterizada segundo a metodologia descrita por Lombardi e col. ${ }^{20}$.

Amostra representativa dos 5.249 nascidos vivos e moradores da zona urbana foi selecionada ao longo do ano por sorteio sistemático seguindo a ordem de nascimento. O tamanho da amostra estudada deveria permitir detectar um risco relativo de, pelo menos, 1,4, com um poder de $90 \%$, sendo acrescidos $5 \%$ para eventuais perdas, resultando em 655 crianças. Essas foram visitadas em seus domicílios quando completavam um e três meses de vida. Questionários padronizados e pré-codificados foram utilizados para coleta de informações sobre incidência, padrões e duração da amamentação, época de introdução de suplementos alimentares na dieta infantil, uso de $\operatorname{chupetas}^{36} \mathrm{e}$ separação mãe-filho nos primeiros dias de vida.

Para a análise bivariada utilizou-se o teste de qui-qua- 
drado de Pearson para diferenças entre proporções e teste-F para diferenças entre médias. Para a análise da duração da amamentação, utilizou-se a técnica de "análise de sobrevivência" e teste "logrank", que corresponde a um teste de qui-quadrado ${ }^{19}$. Para a análise multivariada de proporções utilizou-se a regressão logística ${ }^{8}$ e, para a análise multivariada da duração da amamentação, a regressão de $\mathrm{Cox}^{5}$. A medida de efeito utilizada foi a razão de odds, expressando o excesso de risco - ou probabilidade - de interrupção da lactação de acordo com as categorias de cada variável.

As seguintes definições foram utilizadas:

- Amamentação - Leite materno acompanhado ou não de quaisquer outros alimentos, sólidos ou líquidos, inclusive leites artificiais ${ }^{38}$.

- Amamentação predominante - Leite materno como principal fonte nutritiva, acompanhado ou não de água, chás ou suco de frutas ${ }^{38}$.

-Amamentação exclusiva - Leite materno como única fonte nutritiva ${ }^{38}$.

- Cesariana eletiva - Realizada para ligadura tubária, porque a mulher já havia sido submetida a uma cesariana ou por conveniência da parturiente ou do médico.

- Cesariana emergencial - Realizada por sofrimento fetal, distócias de apresentação, hemorragia materna, parada de progressão, eclâmpsia, pós-datismo, diabete materna (macrossomia fetal) e outros problemas de saúde materna.

\section{RESU LTAD O S}

Foi possível obter informação completa para 649 crianças no primeiro mês e para 644 no terceiro. Nove dos onze casos perdidos mudaram de cidade e duas famílias recusaram-se a participar dos acompanhamentos. As características socioeconômicas, demográficas e biológicas da amostra foram semelhantes às da população de nascidos vivos na zona urbana na cidade de Pelotas, no ano de 1993 (Tabela 1).

Os partos por cesariana representaram $28 \%$ dos nascimentos da amostra, e a maioria teve caráter emergencial (Tabela 2).

Quase todas as crianças - 98\% - iniciaram o aleitamento. As nascidas por cesariana eletiva apresentaram uma incidência de amamentação ligeiramente

Tabela 1 - Características da amostra coorte dos nascidos em Pelotas em 1993 ( $n=655)$.

\begin{tabular}{|c|c|c|}
\hline Característica & $\mathrm{N}$ & $\%(*)$ \\
\hline \multicolumn{3}{|l|}{ Classe social } \\
\hline Burguesia & 5 & $1 \%$ \\
\hline Nova pequena burguesia & 28 & $5 \%$ \\
\hline Pequena burguesia tradicional & 104 & $17 \%$ \\
\hline Proletariado não típico & 283 & $46 \%$ \\
\hline Proletariado típico & 137 & $23 \%$ \\
\hline Subproletariado & 49 & $8 \%$ \\
\hline \multicolumn{3}{|l|}{ Educação materna (anos completos) } \\
\hline 9 & 169 & $26 \%$ \\
\hline $5-8$ & 314 & $48 \%$ \\
\hline $0-4$ & 172 & $26 \%$ \\
\hline \multicolumn{3}{|l|}{ Idade materna (anos completos) } \\
\hline$<20$ & 97 & $15 \%$ \\
\hline $20-24$ & 194 & $30 \%$ \\
\hline $25-29$ & 179 & $27 \%$ \\
\hline $30-34$ & 119 & $18 \%$ \\
\hline$\geq 35$ & 66 & $10 \%$ \\
\hline \multicolumn{3}{|l|}{ Paridade ( $n \circ$ de filhos) } \\
\hline 0 & 223 & $34 \%$ \\
\hline 1 & 186 & $28 \%$ \\
\hline 2 & 128 & $20 \%$ \\
\hline$\geq 3$ & 118 & $18 \%$ \\
\hline \multicolumn{3}{|l|}{ Número de consultas pré-natais } \\
\hline$\geq 10$ & 185 & $28 \%$ \\
\hline $5-9$ & 374 & $57 \%$ \\
\hline $1-4$ & 64 & $10 \%$ \\
\hline 0 & 32 & $5 \%$ \\
\hline Experiência materna prévia com lactação (já amamentaram & 372 & $57 \%$ \\
\hline Baixo peso ao nascer $(<2.500 \mathrm{~g})$ & 53 & $8 \%$ \\
\hline Prematuridade (Dubowitz) & 48 & $7 \%$ \\
\hline
\end{tabular}


menor, mas esta diferença não foi estatisticamente significativa (Tabela 2).

Crianças recebendo exclusivamente leite materno nesta amostra foram raras: 99 (15\%) aos 30 dias de vida e $43(7 \%)$ aos 90 dias, não diferindo entre os tipos de parto.

Observaram-se diminuições importantes nas prevalências de amamentação (de $87 \%$ para $59 \%$ ) e amamentação predominante (de $61 \%$ para $28 \%$ ) entre o primeiro e o terceiro mês de vida, mas essas prevalências não estiveram significativamente associadas ao tipo de parto na análise bivariada.

Testou-se a associação com possíveis fatores de confusão. As variáveis "classe social" e "educação materna" foram consideradas "a priori" como possíveis fatores de confusão sobredeterminantes dos demais fenômenos em estudo, apesar de não se apresentarem significativamente associadas com amamentação no terceiro mês de vida. $\mathrm{Na}$ análise de prevalência de amamentação, quando controlados os fatores de confusão, observou-se que os nascidos por cesarianas eletivas apresentaram três vezes mais chance de haverem interrompido completamente a lactação aos 30 dias de vida (Razão de odds=3.1, 95\% IC 1.3-7.2) (Tabela 3).

Tabela 2 - Freqüência de nascimentos e incidência de amamentação segundo o tipo de parto. Coorte dos nascidos em Pelotas em 1993.

\begin{tabular}{lrrrr}
\hline Tipo de parto & \multicolumn{2}{c}{$\begin{array}{c}\text { Freqüência de } \\
\text { nascimentos }\end{array}$} & \multicolumn{2}{c}{ Incidência de } \\
& N & $\%$ & N & $\%$ \\
\hline Vaginal & 468 & 72 & 459 & 98 \\
Cesariana eletiva & 52 & 8 & 49 & 94 \\
Cesariana emergencial & 135 & 20 & 132 & 98 \\
\hline Total & 655 & 100 & 640 & 98 \\
\hline
\end{tabular}

A análise bivariada da duração da amamentação, utilizando técnicas de sobrevivência não mostrou associação com os tipos de parto (Figura 1). Entretanto, quando controlados os fatores de confusão, evidenciaram-se algumas diferenças. A exemplo da análise de proporções, as crianças nascidas por cesariana eletiva tiveram risco cerca de três vezes maior de terem sido desmamadas ao final do primeiro mês do que as nascidas por parto vaginal ou cesariana emergencial (Razão de odds $=2,6,95 \%$ IC 1,3-5,3). Observou-se ainda que esta diferença se manteve significativa somente entre o $20^{\circ}$ e o $40^{\circ}$ dias de vida. Do $40^{\circ}$ ao $90^{\circ}$ dia de vida a diferença entre os riscos não alcançou significância estatística (Figuras 2 e 3).

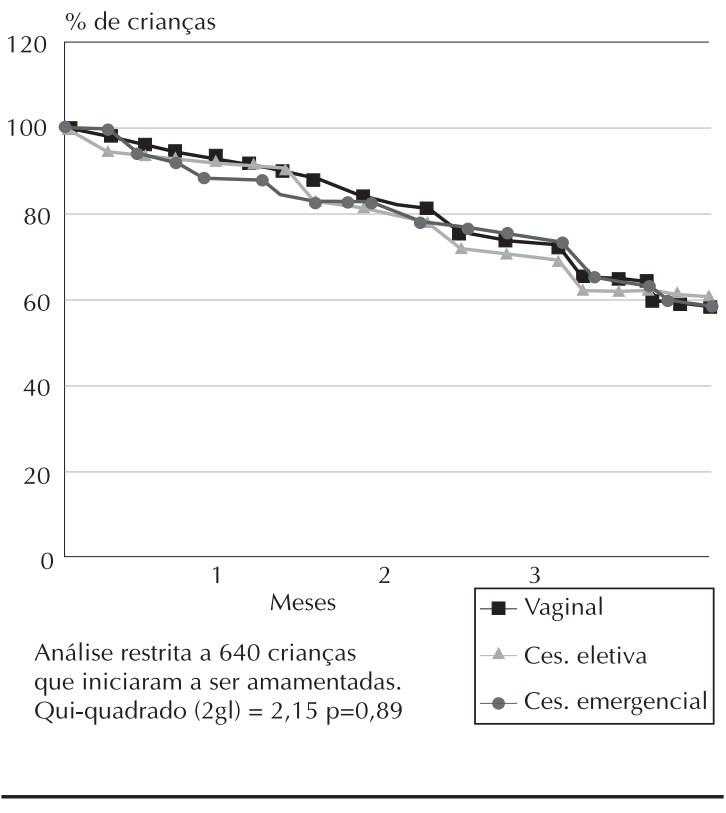

Figura 1- Duração da amamentação conforme o tipo de parto. Pelotas, RS, 1993.

Tabela 3 - Razão de "O dds" de Prevalência (RO P e 95\% IC) para desmame segundo o tipo de parto. Acompanhamento do primeiro e terceiro meses de vida da coorte dos nascidos em Pelotas em 1993 ( $n=655)$.

\begin{tabular}{lcccc}
\hline \multirow{2}{*}{ Tipo de parto } & \multicolumn{3}{c}{ ROP para desmame } \\
\cline { 2 - 5 } & \multicolumn{2}{c}{300 dia de vida } & \multicolumn{2}{c}{90 0 dia de vida } \\
\cline { 2 - 5 } & Bruto (IC95\%) & Ajustado*(IC95\%) & Bruto (IC95\%) & Ajustado**(IC95\%) \\
\hline Vaginal & $1,00-$ & $1,00-$ & $1,00-$ & 1,00 \\
Cesariana Eletiva & $1,82(0,89-3,74)$ & $3,09(1,32-7,23)$ & $0,87(0,48-1,58)$ & $1,31(0,68-2,52)$ \\
Cesariana Emergencial & $0,98(0,55-1,74)$ & $0,86(0,46-1,62)$ & $0,93(0,63-1,38)$ & $0,81(0,53-1,24)$ \\
& TRV $(2 \mathrm{gl}) 2,57$ & $0,86(0,46-1,62)$ & TRV $(2 \mathrm{gl}) 0,28$ & TRV $(2 \mathrm{gl}) 1,82$ \\
& $\mathrm{p}=0,28$ & $\mathrm{p}=0,03$ & $\mathrm{p}=0,87$ & $\mathrm{p}=0,40$ \\
\hline
\end{tabular}

TRV: teste de razão de verossimilhança.

* Ajuste para: classe social, escolaridade, idade, paridade, experiência prévia com lactação, internações na gestação, peso ao nascer, idade gestacional e morbidade do recém-nascido.

** Ajuste para: classe social, escolaridade, idade, paridade, experiência prévia com lactação, pré-natal, peso ao nascer, idade gestacional, alta hospitalar não simultânea 


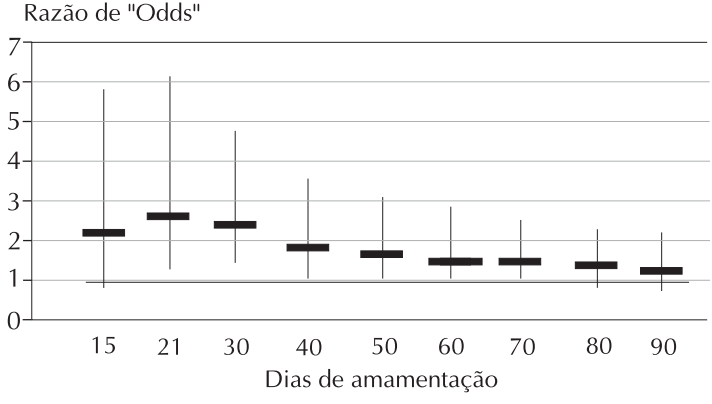

Razão de "Odds" = 1 parto vaginal

Figura 2 - Estimativa do risco relativo (razão de "O dds" e 95\% IC para interrupção da amamentação em cesarianas eletivas. Pelotas, RS, 1993.

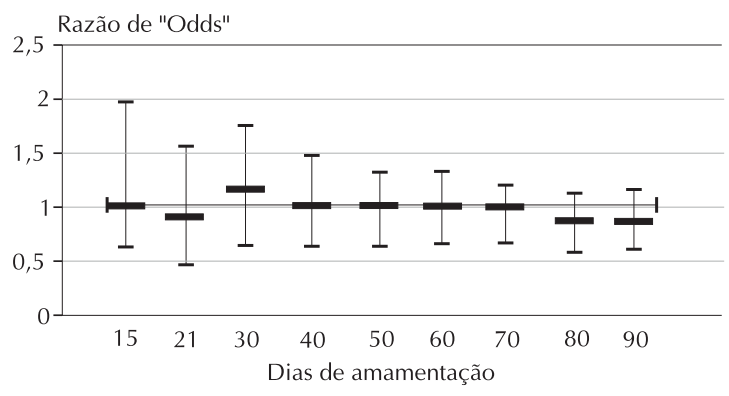

Razão de "Odds" = 1 parto vaginal

Figura 3 - Estimativa do risco relativo (razão de "O dds") e 95\% IC para interrupção da amamentação em cesarianas emergenciais. Pelotas, RS, 1993.

\section{DISCU SSÃO}

Os resultados mostraram que não houve diferenças nas incidências de amamentação conforme o tipo de parto, tendo sido consistentes com outros dois estudos já realizados no Brasil ${ }^{4,35}$. A prevalência de crianças com amamentação predominante aos 30 e 90 dias de vida também não diferiu conforme o tipo de parto, sendo preocupantemente baixa em todos os grupos, se comparada com o recomendado pela Organização Mundial da Saúde ${ }^{3,13}$.

Mães submetidas a cesarianas eletivas, quando comparadas àquelas que tiveram partos vaginais ou cesarianas emergenciais, apresentaram um risco aumentado de interrupção completa da lactação no primeiro mês de vida. Este achado evidenciou-se apenas na análise multivariada, quando controlados os fatores de confusão.

Estes resultados podem ter sido afetados pelos métodos utilizados, principalmente pela classificação das cesarianas em eletivas ou emergenciais. Tal classificação, apesar de baseada em um critério arbitrário e na informação fornecida pelas mães, iden- tificou grupos com padrões diferentes de amamentação. Prováveis erros de classificação teriam levado cesarianas eletivas a serem classificadas como emergenciais, implicando numa subestimativa das diferenças (aproximação da razão de odds de prevalência da unidade no grupo nascido por cesarianas eletivas $)^{35}$.

Os dados contrastam com os de estudos que descreveram as cesarianas como fator de risco para não iniciar a lactação ou interrompê-la nos primeiros dias de vida. É possível que variações das práticas hospitalares expliquem estas diferenças. Assim, hospitais cujo padrão de atendimento pós-operatório dificulte o alojamento conjunto e o aleitamento à livre-demanda, ou ainda permitam a introdução precoce de outros alimentos na dieta infantil, poderiam levar a um maior insucesso do aleitamento.

Os dados contrastam também com os de estudos que descrevem não haver associação entre tipo de parto e duração da amamentação. Esses estudos podem ter seus resultados devidos a problemas metodológicos já abordados, principalmente à presença de fatores de confusão com efeitos independentes sobre a amamentação, bem como a diferenças nos padrões de risco para interrupção da lactação entre as populações estudadas.

O único estudo identificado na literatura que diferencia cesarianas eletivas e emergenciais foi realizado na mesma área geográfica deste, com mulheres que tiveram seus filhos em 1982. Naquela amostra não houve diferenças na incidência de amamentação conforme o tipo de parto, mas um aumento de risco de interrupção da lactação aos seis meses para os nascidos por cesarianas emergenciais ${ }^{35}$. A distribuição de nascimentos conforme o tipo de parto não diferiu nesses dois estudos, o que sugere que os padrões de realização de cesarianas na região não se modificaram na última década, mas sim o padrão de aleitamento.

Apesar de não ter sido evidenciada uma associação estatisticamente significativa entre tipo de parto e incidência e duração da amamentação, parece possível que mães que se submetem a cesarianas eletivas possam ser diferentes das demais em aspectos comportamentais também associados à amamentação. Tais características são de difícil captação pelo método epidemiológico e configurariam um viés de auto-seleção. Provavelmente o tempo de hospitalização diferenciado altere práticas hospitalares relacionadas à amamentação, fazendo com que mães cesariadas recebam menos estímulo ao aleitamento durante o pós-operatório.

O fato da incidência e duração da lactação terem 
sido similares entre os nascidos por partos vaginais e cesarianas emergenciais não deve ser razão de aceitação das elevadas taxas de cesarianas no Brasil ${ }^{35}$.

Sugere-se que outros estudos englobem um componente etnográfico, a fim de avaliar qualitativamente os efeitos de um possível viés de auto-seleção entre as parturientes submetidas a cesarianas eletivas. O estudo da diversidade de padrões de crescimento e níveis atuais de cesarianas, bem como os motivos para sua realização em subgrupos populacionais, oferecerá oportunidade para uma maior compreensão dos fatores que determinam as diferenças nesta prática obstétrica, ainda a ser explorado no Brasil. Juntamente com a investigação cuidadosa de tendências internacionais para motivos de realização de cesarianas, poder-se-á identificar padrões passíveis de in-

\section{REFERÊNCIAS}

1. BARROS, F.C.; TEMER, T.C.; TONIOLI FILHO, S.; TOMASI, E.; VICTORA, C.G. The impact of lactation centres on breastfeeding patterns, morbidity and growth: a birth cohort study. Acta Paediatr., 84:1221-6, 1995.

2. BARROS, F.C.; VAUGHAN, J.P.; VICTORA, C.G. Why so many caesarean sections? The need for a further policy change in Brazil. Health Policy Plann., 1:19-29,1986.

3. BARROS, F.C.; VAUGHAN, J.P.; VICTORA, C.G.; HUTTLY, S.R.A. Epidemic of cesarean sections in Brazil. Lancet, 338:167-9,1991.

4. BARROS, F.C. \& VICTORA,C.G. Breast-feeding and diarrhea in Brazilian children. New York, DHS, 1990. (Demographic and Health Surveys. Futher Analysis Series, 3).

5. COX, D.R. \& OAKES, D. Analysis of survival data. New York, Chapman and Hall, 1984.

6. DUBOWITZ, L.M.S.; DUBOWITZ, V.; GOLDBERG, C. Clinical assessment of gestacional age in the newborn infant. J.Pediatr., 77:1-10,1970.

7. ELLIS, D. \& HEWAT, R. Factors related to breastfeeding duration. Can.Fam.Physician, 30:1479-84,1984.

8. EGRET. EGRET user's manual. Washington,Statistics and Epidemiology Research Corporation, 1988.

9. EVER-HADANI, P.; SEIDMAN, D.S.; MANOR, O.; HARLAP, S. Breast feeding in Israel: maternal factors associated with choice and duration. $J$. Epidemiol.Community Health, 48:281-5,1994.

10. FAÚNDES, A. \& CECATTI, J.G. Which policy for cesarean sections in Brazil? An analysis of trends and consequences. Health Policy Plann., 8:33-42,1994.

11. FORMAN, M.R.; BERENDEST, H.W.; LEWANDO- tervenções que diminuíssem sua elevada ocorrência.

Recomenda-se aos serviços de saúde que destinem especial atenção à lactantes submetidas a cesarianas eletivas, com o objetivo de promover o aleitamento desde as primeiras semanas de vida, evitando assim sua interrupção precoce.

Além disso, é necessário que a promoção do aleitamento materno seja feita de forma mais efetiva, para que se eleve a proporção de crianças exclusivamente amamentadas no primeiro trimestre de vida, independente do tipo de parto. Medidas como a capacitação de trabalhadores em serviços de saúde para o manejo clínico da lactação em centros de referência ${ }^{1}$, além da utilização da mídia para campanhas de massa poderiam ser adotadas em favor da promoção da amamentação.
HUNDT, G.; SORAV, B.; NAGGAN, L. Perinatal factors influencing infant feeding practices at birth: the Bedouin Infant Feeding Study. Paediatr.Perinat. Epidemiol., 5:16880,1991 .

12. GATHWALA, G. \& NARAYANAN, I. Delayed contact and breast feeding. Indian Pediatr., 29:155-9,1992.

13. GIUGLIANI, E.R. Amamentação: como e por que promover. J. Pediatr., 70:138-51,1994.

14. GOULD, J.B.; DAVEY, B.; STAFFORD, R.S. Socioeconomic differences in rates of caesarian section. N.Engl.J.Med., 321:233-9,1989.

15. GULICK, E.E. Informational correlates of successful breastfeeding. Matern. Child Nurs. J., 7:370-5,1982.

16. JANKE, J.R. Breastfeeding duration following cesarean and vaginal births. J.Nurse Midwifery, 33:133-64,1988.

17. JANOWITZ, B.; NAKAMURA, M.S.; LINS, F.E.; BROWN, M.L.; CLOPTON, D. Cesarean section in Brazil. Soc.Sci.Med., 16:19-25,1982.

18. KEARNEY, M.; CRONEWETT, L.R.; REINHARDT, R. Cesarean delivery and breast-feeding outcomes. Birth, 17:97-103,1990.

19. KIRKWOOD, B. Essentials of medical statistics. London, Blackwell Scientific, 1989.

20. LOMBARDI, C.; BRONFMAN, M.; FACCHINI, L.A.; VICTORA, C.G.; BARROS, F.C.; BERIA, J.U.; TEIXEIRA, A.M. Operacionalização do conceito de classe social em estudos epidemiológicos. Rev.Saúde Pública, 22:253-65, 1988.

21. LOUGHLIN, H.H.; CLAPP-CHANNING, N.E.; GEHLBACH, S.H.; POLLARD, J.C.; MCCUTCHEN, T.M. 
Early termination of breast-feeding: identifying those at risk. Pediatrics, 75:508-13,1985.

22. MANSBACH, I.K.; GREENBAUM, C.W.; SULKES, J. Onset and duration of breast feeding among Israeli mothers: relationships with smoking and type of delivery. Soc.Sci.Med., 33:1391-7,1991.

23. MARTINES, J.C.; ASHWORTH, A.; KIRKWOOD, B Breast-feeding among the urban poor in southern Brazil: reasons for termination in the first 6 months of life. Bull.World Health Organ., 67:151-61,1989.

24. NOTZON, F.C.; PLACEK, P.J.; TAFFEL, S.M. Comparisons of National caesarean-section rates. N.Engl.J.Med., 316:386-9, 1987 .

25. NOTZON, F.C.; CNATTINGIUS, S.; BERGSJÖ, P.; COLE, S.; TAFFEL, S.; IRGENS, L.; DALTVEIT, A.K. Caesarean section delivery in the 1980s: international comparison by indication. Am.J.Obstet. Gynecol., 170:495-504,1994.

26. PROCIANOY, R.S.; FERNANDES-FILHO, P.H.; LAZARO, L.; SARTORI, N. Factors affecting breastfeeding: the influence of caesarean section. J.Trop.Pediatr. 30:39-42,1984.

27. ROMERO-GWYNN, E. \& CARIAS, L. Breast-feeding intentions and practice among hispanic mothers in Southern California. Pediatrics, 84:626-32,1989.

28. SAMUELS, E.S.; MARGEN, S.; SCHOEN, E.J. Incidence and duration of breast-feeding in a health maintenance organization population. Am.J.Clin.Nutr., 2:504-10,1985

29. SCRIMSHAW, S.C.M.; ENGLE, P.L.; ARNOLD, L.; HAYNES, K. Factors affecting breast-feeding among women of Mexican origin or descendents in Los Angeles. Am.J.Public Health, 74:467-70,1987.

30. STEPHENSON, P.A.; BAKOULA, C.; HEMMINKI, E.; KNUDSEN, L.; LEVASSEUR,M.S.; CHENKER, J.; STEMBERA, Z.; TIBA, J.; VERBRUGGE, H.P.; ZUPAN, J.; WAGNER, M.G.; KARAGAS, M.; PIZACANI,
B.; PINEAULT, R.; TUIMALA, R.; HOUD, S.; LOMAS, J. Patterns of use of obstetrical interventions in 12 countries. Pediatr. Perinat. Epidemiol., 7:45-54,1993.

31. TAMMINEN, T.; VERRONEEN, P.; SAARIKOSKI, S.; GÖRANSSON, A.; TUOMIRANTA, H. The influence of perinatal factors on breast-feeding. Acta Paediatr.Scand., 72:9-12,1983

32. TROWELL, J. Possible effects of emergency caesarian section on the mother-child relationship. Early Hum.Dev., 7:41-51,1982.

33. VESTERMARK, V.; HOGDALL, C.K.; BIRCH, M.; PLENOV, G.; TOFTAGER-ARSEN, K. Influence of the mode of delivery on iniciation of breast-feeding. Eur.J.Obstet.Gynecol.Reprod.Biol., 38:33-8, 1990.

34. VICTORA, C.G.; HUTTLY, S.R.A.; BARROS, F.C.; VAUGHAN, J.P. Caesarean section and duration of breast feeding among Brazilians. Arch.Dis.Child., 65:632-4,1990.

35. VICTORA, C.G.; FACCHINI, L.A.; BARROS, F.C.; LOMBARDI, C. Pobreza e saúde: como medir nível socioeconômico em estudos epidemiológicos de saúde infantil? In: Congresso Brasileiro de Epidemiologia, $1^{\circ}$, Campinas, 1990. Anais. Campinas, 1992. p. 302-14.

36. VICTORA, C.G.; TOMASI, E.; OLINTO, M.T.A.; BARROS, F.C. Use of pacifiers and breastfeeding duration. Lancet, 341:404-6,1993.

37. WICHELOW, M. Factors associated with the duration of breast-feeding in a privileged society. Early Hum.Dev. 7:273-80,1982.

38. WORLD HEALTH ORGANIZATION. Division of Diarrheal and Acute Respiratory Disease Control. Indicators for assessing breast-feeding practices: report of an informal meeting. Geneva, 1991.

39. WRIGHT, H.J. \& WALKER, P.C. Prediction of duration of breast-feeding in primiparas. J.Epidemiol.Community Health, 37:89-94,1983. 